\title{
Distributed Representations of Geographically Situated Language
}

\author{
David Bamman Chris Dyer Noah A. Smith \\ School of Computer Science \\ Carnegie Mellon University \\ Pittsburgh, PA 15213, USA \\ \{dbamman, cdyer, nasmith\}@es.cmu.edu
}

\begin{abstract}
We introduce a model for incorporating contextual information (such as geography) in learning vector-space representations of situated language. In contrast to approaches to multimodal representation learning that have used properties of the object being described (such as its color), our model includes information about the subject (i.e., the speaker), allowing us to learn the contours of a word's meaning that are shaped by the context in which it is uttered. In a quantitative evaluation on the task of judging geographically informed semantic similarity between representations learned from 1.1 billion words of geo-located tweets, our joint model outperforms comparable independent models that learn meaning in isolation.
\end{abstract}

\section{Introduction}

The vast textual resources used in NLP newswire, web text, parliamentary proceedings can encourage a view of language as a disembodied phenomenon. The rise of social media, however, with its large volume of text paired with information about its author and social context, reminds us that each word is uttered by a particular person at a particular place and time. In short: language is situated.

The coupling of text with demographic information has enabled computational modeling of linguistic variation, including uncovering words and topics that are characteristic of geographical regions (Eisenstein et al., 2010; O'Connor et al., 2010; Hong et al., 2012; Doyle, 2014), learning correlations between words and socioeconomic variables (Rao et al., 2010; Eisenstein et al., 2011; Pennacchiotti and Popescu, 2011; Bamman et al., 2014); and charting how new terms spread geographically (Eisenstein et al., 2012). These models can tell us that hella was (at one time) used most often by a particular demographic group in northern California, echoing earlier linguistic studies (Bucholtz, 2006), and that wicked is used most often in New England (Ravindranath, 2011); and they have practical applications, facilitating tasks like text-based geolocation (Wing and Baldridge, 2011; Roller et al., 2012; Ikawa et al., 2012). One desideratum that remains, however, is how the meaning of these terms is shaped by geographical influences - while wicked is used throughout the United States to mean bad or evil ("he is a wicked man"), in New England it is used as an adverbial intensifier ("my boy's wicked smart"). In leveraging grounded social media to uncover linguistic variation, what we want to learn is how a word's meaning is shaped by its geography.

In this paper, we introduce a method that extends vector-space lexical semantic models to learn representations of geographically situated language. Vector-space models of lexical semantics have been a popular and effective approach to learning representations of word meaning (Lin, 1998; Turney and Pantel, 2010; Reisinger and Mooney, 2010; Socher et al., 2013; Mikolov et al., 2013 , inter alia). In bringing in extra-linguistic information to learn word representations, our work falls into the general domain of multimodal learning; while other work has used visual information to improve distributed representations (Andrews et al., 2009; Feng and Lapata, 2010; Bruni et al., 2011; Bruni et al., 2012a; Bruni et al., 2012b; Roller and im Walde, 2013), this work generally exploits information about the object being described (e.g., strawberry and a picture of a strawberry); in contrast, we use information about the speaker to learn representations that vary according to contextual variables from the speaker's perspective. Unlike classic multimodal systems that incorporate multiple active modalities (such as gesture) from a user (Oviatt, 2003; Yu and 


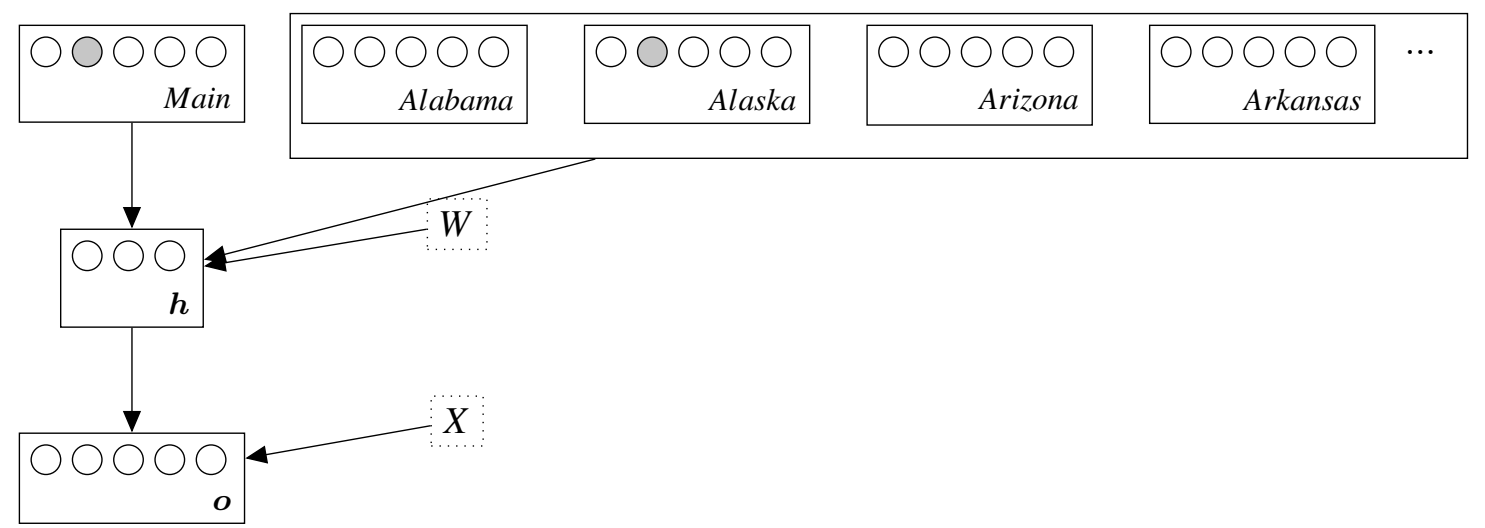

Figure 1: Model. Illustrated are the input dimensions that fire for a single sample, reflecting a particular word (vocabulary item \#2) spoken in Alaska, along with a single output. Parameter matrix $W$ consists of the learned low-dimensional embeddings.

Ballard, 2004), our primary input is textual data, supplemented with metadata about the author and the moment of authorship. This information enables learning models of word meaning that are sensitive to such factors, allowing us to distinguish, for example, between the usage of wicked in Massachusetts from the usage of that word elsewhere, and letting us better associate geographically grounded named entities (e.g, Boston) with their hypernyms (city) in their respective regions.

\section{Model}

The model we introduce is grounded in the distributional hypothesis (Harris, 1954), that two words are similar by appearing in the same kinds of contexts (where "context" itself can be variously defined as the bag or sequence of tokens around a target word, either by linear distance or dependency path). We can invoke the distributional hypothesis for many instances of regional variation by observing that such variants often appear in similar contexts. For example:

- my boy's wicked smart

- my boy's hella smart

- my boy's very smart

Here, all three variants can often be seen in an immediately pre-adjectival position (as is common with intensifying adverbs).

Given the empirical success of vector-space representations in capturing semantic properties and their success at a variety of NLP tasks (Turian et al., 2010; Socher et al., 2011; Collobert et al., 2011; Socher et al., 2013), we use a simple, but state-of-the-art neural architecture (Mikolov et al., 2013) to learn low-dimensional real-valued repre- sentations of words. The graphical form of this model is illustrated in figure 1 .

This model corresponds to an extension of the "skip-gram" language model (Mikolov et al., 2013) (hereafter SGLM). Given an input sentence $s$ and a context window of size $t$, each word $s_{i}$ is conditioned on in turn to predict the identities of all of the tokens within $t$ words around it. For a vocabulary $V$, each input word $s_{i}$ is represented as a one-hot vector $\boldsymbol{w}_{i}$ of length $|V|$. The SGLM has two sets of parameters. The first is the representation matrix $W \in \mathbb{R}^{|V| \times k}$, which encodes the real-valued embeddings for each word in the vocabulary. A matrix multiply $\boldsymbol{h}=\boldsymbol{w}^{\top} W, \in \mathbb{R}^{k}$ serves to index the particular embedding for word $\boldsymbol{w}$, which constitutes the model's hidden layer. To predict the value of the context word $y$ (again, a one-hot vector of dimensionality $|V|$ ), this hidden representation $h$ is then multiplied by a second parameter matrix $X \in \mathbb{R}^{|V| \times k}$. The final prediction over the output vocabulary is then found by passing this resulting vector through the softmax function $\boldsymbol{o}=\operatorname{softmax}(X \boldsymbol{h})$, giving a vector in the $|V|$ dimensional unit simplex. Backpropagation using (input $x$, output $y$ ) word tuples learns the values of $W$ (the embeddings) and $X$ (the output parameter matrix) that maximize the likelihood of $y$ (i.e., the context words) conditioned on $x$ (i.e., the $s_{i}$ 's). During backpropagation, the errors propagated are the difference between $\boldsymbol{o}$ (a probability distribution with $k$ outcomes) and the true (one-hot) output $y$.

Let us define a set of contextual variables $\mathcal{C}$; in the experiments that follow, $\mathcal{C}$ is comprised solely of geographical state $\mathcal{C}_{\text {state }}=$ $\{\mathrm{AK}, \mathrm{AL}, \ldots, \mathrm{WY}\}$ ) but could in principle include any number of features, such as calendar 
month, day of week, or other demographic variables of the speaker. Let $|\mathcal{C}|$ denote the sum of the cardinalities of all variables in $\mathcal{C}$ (i.e., 51 states, including the District of Columbia). Rather than using a single embedding matrix $W$ that contains low-dimensional representations for every word in the vocabulary, we define a global embedding matrix $W_{\text {main }} \in \mathbb{R}^{|V| \times k}$ and an additional $|\mathcal{C}|$ such matrices (each again of size $|V| \times k$, which capture the effect that each variable value has on each word in the vocabulary. Given an input word $\boldsymbol{w}$ and set of active variable values $\mathcal{A}$ (e.g., $\mathcal{A}=$ $\{$ state $=\mathrm{MA}\}$ ), we calculate the hidden layer $\boldsymbol{h}$ as the sum of these independent embeddings: $\boldsymbol{h}=\boldsymbol{w}^{\top} W_{\text {main }}+\sum_{a \in \mathcal{A}} \boldsymbol{w}^{\top} W_{a}$. While the word wicked has a common low-dimensional representation in $W_{\text {main,wicked }}$ that is invoked for every instance of its use (regardless of the place), the corresponding vector $W_{\mathrm{MA} \text {, wicked }}$ indicates how that common representation should shift in $k$ dimensional space when used in Massachusetts. Backpropagation functions as in standard SGLM, with gradient updates for each training example $\{x, y\}$ touching not only $W_{\text {main }}$ (as in SGLM), but all active $W_{\mathcal{A}}$ as well.

The additional $W$ embeddings we add lead to an increase in the number of total parameters by a factor of $|\mathcal{C}|$. To control for the extra degrees of freedom this entails, we add squared $\ell_{2}$ regularization to all parameters, using stochastic gradient descent for backpropagation with minibatch updates for the regularization term. As in Mikolov et al. (2013), we speed up computation using the hierarchical softmax (Morin and Bengio, 2005) on the output matrix $X$.

This model defines a joint parameterization over all variable values in the data, where information from data originating in California, for instance, can influence the representations learned for Wisconsin; a naive alternative would be to simply train individual models on each variable value (a "California" model using data only from California, etc.). A joint model has three a priori advantages over independent models: (i) sharing data across variable values encourages representations across those values to be similar; e.g., while city may be closer to Boston in Massachusetts and Chicago in Illinois, in both places it still generally connotes a municipality; (ii) such sharing can mitigate data sparseness for less-witnessed areas; and (iii) with a joint model, all representations are guaranteed to be in the same vector space and can therefore be compared to each other; with individual models (each with different initializations), word vectors across different states may not be directly compared.

\section{Evaluation}

We evaluate our model by confirming its face validity in a qualitative analysis and estimating its accuracy at the quantitative task of judging geographically-informed semantic similarity. We use 1.1 billion tokens from 93 million geolocated tweets gathered between September 1, 2011 and August 30, 2013 (approximately 127,000 tweets per day evenly sampled over those two years). This data only includes tweets that have been geolocated to state-level granularity in the United States using high-precision pattern matching on the user-specified location field (e.g., "new york ny" $\rightarrow$ NY, "chicago" $\rightarrow$ IL, etc.). As a preprocessing step, we identify a set of target multiword expressions in this corpus as the maximal sequence of adjectives + nouns with the highest pointwise mutual information; in all experiments described below, we define the vocabulary $V$ as the most frequent 100,000 terms (either unigrams or multiword expressions) in the total data, and set the dimensionality of the embedding $k=100$. In all experiments, the contextual variable is the observed US state (including DC), so that $|\mathcal{C}|=51$; the vector space representation of word $\boldsymbol{w}$ in state $s$ is $\boldsymbol{w}^{\top} W_{\text {main }}+\boldsymbol{w}^{\top} W_{s}$.

\subsection{Qualitative Evaluation}

To illustrate how the model described above can learn geographically-informed semantic representations of words, table 1 displays the terms with the highest cosine similarity to wicked in Kansas and Massachusetts after running our joint model on the full 1.1 billion words of Twitter data; while wicked in Kansas is close to other evaluative terms like evil and pure and religious terms like gods and spirit, in Massachusetts it is most similar to other intensifiers like super, ridiculously and insanely.

Table 2 likewise presents the terms with the highest cosine similarity to city in both California and New York; while the terms most evoked by city in California include regional locations like Chinatown, Los Angeles' South Bay and San Francisco's East Bay, in New York the most similar terms include hamptons, upstate and borough 


\begin{tabular}{|l|c||l|c|}
\hline \multicolumn{2}{|c||}{ Kansas } & \multicolumn{2}{c|}{ Massachusetts } \\
\hline term & cosine & term & cosine \\
\hline \hline wicked & 1.000 & wicked & 1.000 \\
\hline evil & 0.884 & super & 0.855 \\
\hline pure & 0.841 & ridiculously & 0.851 \\
\hline gods & 0.841 & insanely & 0.820 \\
\hline mystery & 0.830 & extremely & 0.793 \\
\hline spirit & 0.830 & goddamn & 0.781 \\
\hline king & 0.828 & surprisingly & 0.774 \\
\hline above & 0.825 & kinda & 0.772 \\
\hline righteous & 0.823 & \#sarcasm & 0.772 \\
\hline magic & 0.822 & sooooooo & 0.770 \\
\hline
\end{tabular}

Table 1: Terms with the highest cosine similarity to wicked in Kansas and Massachusetts.

\begin{tabular}{|l|c||l|r|}
\hline \multicolumn{2}{|c||}{ California } & \multicolumn{2}{c|}{ New York } \\
\hline term & cosine & term & cosine \\
\hline \hline city & 1.000 & city & 1.000 \\
\hline valley & 0.880 & suburbs & 0.866 \\
\hline bay & 0.874 & town & 0.855 \\
\hline downtown & 0.873 & hamptons & 0.852 \\
\hline chinatown & 0.854 & big city & 0.842 \\
\hline south bay & 0.854 & borough & 0.837 \\
\hline area & 0.851 & neighborhood & 0.835 \\
\hline east bay & 0.845 & downtown & 0.827 \\
\hline neighborhood & 0.843 & upstate & 0.826 \\
\hline peninsula & 0.840 & big apple & 0.825 \\
\hline
\end{tabular}

Table 2: Terms with the highest cosine similarity to city in California and New York.

(New York City's term of administrative division).

\subsection{Quantitative Evaluation}

As a quantitative measure of our model's performance, we consider the task of judging semantic similarity among words whose meanings are likely to evoke strong geographical correlations. In the absence of a sizable number of linguistically interesting terms (like wicked) that are known to be geographically variable, we consider the proxy of estimating the named entities evoked by specific terms in different geographical regions. As noted above, geographic terms like city provide one such example: in Massachusetts we expect the term city to be more strongly connected to grounded named entities like Boston than to other US cities. We consider seven categories for which we can reasonably expect the connotations of each term to vary by geography; in each case, we calculate the distance between two terms $x$ and $y$ using representations learned for a given state $\left(\delta_{\text {state }}(x, y)\right)$.

1. city. For each state, we measure the distance between the word city and the state's most populous city; e.g., $\delta_{\mathrm{AZ}}($ city, phoenix).

2. state. For each state, the distance between the word state and the state's name; e.g., $\delta_{\mathrm{WI}}($ state, wisconsin).

3. football. For all NFL teams, the distance between the word football and the team name; e.g., $\delta_{\mathrm{IL}}($ football, bears $)$.

4. basketball. For all NBA teams from a US state, the distance between the word basketball and the team name; e.g., $\delta_{\mathrm{FL}}$ (basketball, heat).

5. baseball. For all MLB teams from a US state, the distance between the word baseball and the team name; e.g., $\delta_{\mathrm{IL}}$ (baseball, cubs), $\delta_{\mathrm{IL}}$ (baseball, white sox).

6. hockey. For all NHL teams from a US state, the distance between the word hockey and the team name; e.g., $\delta_{\mathrm{PA}}$ (hockey, penguins).

7. park. For all US national parks, the distance between the word park and the park name; e.g., $\delta_{\mathrm{AK}}($ park, denali $)$.

Each of these questions asks the following: what words are evoked for a given target word (like football)? While football may everywhere evoke similar sports like baseball or soccer or more specific football-related terms like touchdown or field goal, we expect that particular sports teams will be evoked more strongly by the word football in their particular geographical region: in Wisconsin, football should evoke packers, while in Pennsylvania, football evokes steelers. Note that this is not the same as simply asking which sports team is most frequently (or most characteristically) mentioned in a given area; by measuring the distance to a target word (football), we are attempting to estimate the varying strengths of association between concepts in different regions.

For each category, we measure similarity as the average cosine similarity between the vector for the target word for that category (e.g., city) and the corresponding vector for each state-specific answer (e.g., chicago for IL; boston for MA). We compare three different models:

1. JOINT. The full model described in section 2 , in which we learn a global representation for each word along with deviations from that common representation for each state.

2. INDIVIDUAL. For comparison, we also partition the data among all 51 states, and train a single model for each state using only data from that state. In this model, there is no sharing among states; California has the most 


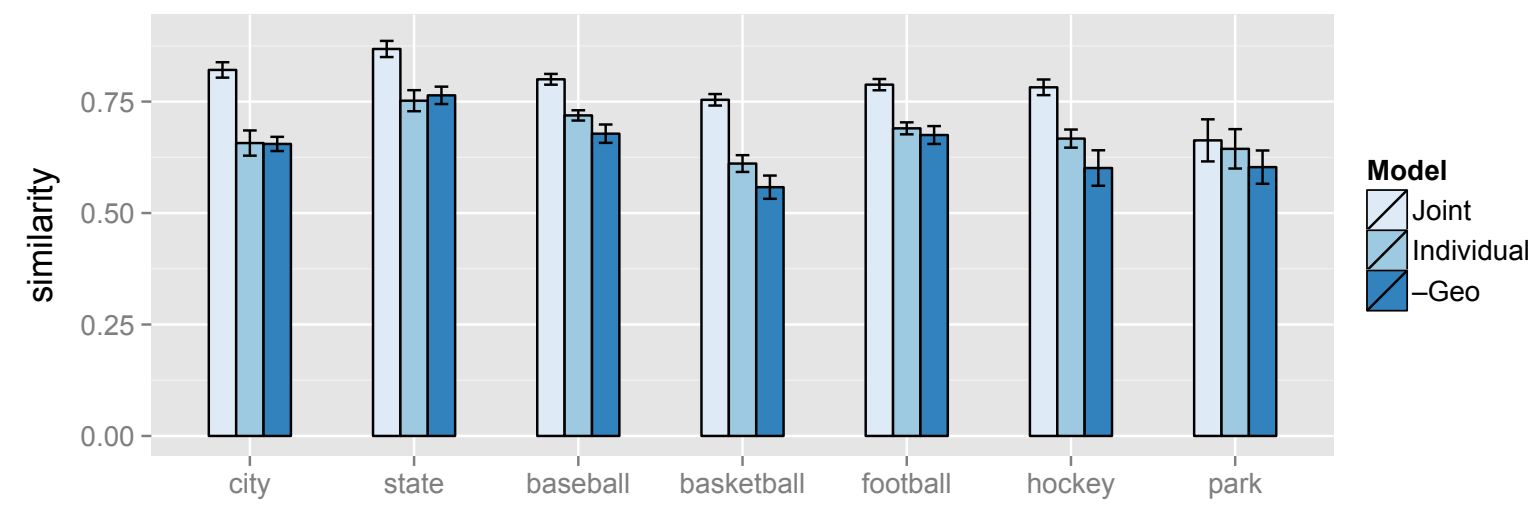

Figure 2: Average cosine similarity for all models across all categories, with $95 \%$ confidence intervals on the mean.

data with 11,604,637 tweets; Wyoming has the least with 47,503 tweets.

3. -GEO. We also train a single model on all of the training data, but ignore any state metadata. In this case the distance $\delta$ between two terms is their overall distance within the entire United States.

As one concrete example of these differences between individual data points, the cosine similarity between city and seattle in the -GEO model is 0.728 (seattle is ranked as the 188th most similar term to city overall); in the INDIVIDUAL model using only tweets from Washington state, $\delta_{W A}($ city, seattle $)=0.780$ (rank \#32); and in the JOINT model, using information from the entire United States with deviations for Washington, $\delta_{W A}($ city, seattle $)=0.858$ (rank \#6). The overall similarity for the city category of each model is the average of 51 such tests (one for each city).

Figure 2 present the results of the full evaluation, including $95 \%$ confidence intervals for each mean. While the two models that include geographical information naturally outperform the model that does not, the JOINT model generally far outperforms the INDIVIDUAL models trained on state-specific subsets of the data. ${ }^{1}$ A model that can exploit all of the information in the data, learning core vector-space representations for all words along with deviations for each contextual variable, is able to learn more geographically-informed representations for this task than strict geographical models alone.

\footnotetext{
${ }^{1}$ This result is robust to the choice of distance metric; an evaluation measuring the Euclidean distance between vectors shows the JOINT model to outperform the INDIVIDUAL and -GEO models across all seven categories.
}

\section{Conclusion}

We introduced a model for leveraging situational information in learning vector-space representations of words that are sensitive to the speaker's social context. While our results use geographical information in learning low-dimensional representations, other contextual variables are straightforward to include as well; incorporating effects for time - such as time of day, month of year and absolute year - may be a powerful tool for revealing periodic and historical influences on lexical semantics.

Our approach explores the degree to which geography, and other contextual factors, influence word meaning in addition to frequency of usage. By allowing all words in different regions (or more generally, with different metadata factors) to exist in the same vector space, we are able compare different points in that space - for example, to ask what terms used in Chicago are most similar to hot dog in New York, or what word groups shift together in the same region in comparison to the background (indicating the shift of an entire semantic field). All datasets and software to support these geographically-informed representations can be found at: http://www.ark. cs. cmu . edu/geoSGLM.

\section{Acknowledgments}

The research reported in this article was supported by US NSF grants IIS-1251131 and CAREER IIS1054319, and by an ARCS scholarship to D.B. This work was made possible through the use of computing resources made available by the Open Cloud Consortium, Yahoo and the Pittsburgh Supercomputing Center. 


\section{References}

Mark Andrews, Gabriella Vigliocco, and David Vinson. 2009. Integrating experiential and distributional data to learn semantic representations. Psychological Review, 116(3):463-498.

David Bamman, Jacob Eisenstein, and Tyler Schnoebelen. 2014. Gender identity and lexical variation in social media. Journal of Sociolinguistics, 18(2).

Elia Bruni, Giang Binh Tran, and Marco Baroni. 2011. Distributional semantics from text and images. In Proc. of the Workshop on Geometrical Models of Natural Language Semantics.

Elia Bruni, Gemma Boleda, Marco Baroni, and NamKhanh Tran. 2012a. Distributional semantics in technicolor. In Proc. of ACL.

Elia Bruni, Jasper Uijlings, Marco Baroni, and Nicu Sebe. 2012b. Distributional semantics with eyes: Using image analysis to improve computational representations of word meaning. In Proc. of the ACM International Conference on Multimedia.

Mary Bucholtz. 2006. Word up: Social meanings of slang in California youth culture. In Jane Goodman and Leila Monaghan, editors, A Cultural Approach to Interpersonal Communication: Essential Readings, Malden, MA. Blackwell.

Ronan Collobert, Jason Weston, Léon Bottou, Michael Karlen, Koray Kavukcuoglu, and Pavel Kuksa. 2011. Natural language processing (almost) from scratch. Journal of Machine Learning Research, 12:2493-2537.

Gabriel Doyle. 2014. Mapping dialectal variation by querying social media. In Proc. of EACL.

Jacob Eisenstein, Brendan O'Connor, Noah A. Smith, and Eric P. Xing. 2010. A latent variable model for geographic lexical variation. In Proc. of EMNLP.

Jacob Eisenstein, Noah A. Smith, and Eric P. Xing. 2011. Discovering sociolinguistic associations with structured sparsity. In Proc. of ACL.

Jacob Eisenstein, Brendan O'Connor, Noah A. Smith, and Eric P. Xing. 2012. Mapping the geographical diffusion of new words. arXiv, abs/1210.5268.

Yansong Feng and Mirella Lapata. 2010. Visual information in semantic representation. In Proc. of NAACL.

Zellig Harris. 1954. Distributional structure. Word, 10(23):146-162.

Liangjie Hong, Amr Ahmed, Siva Gurumurthy, Alexander J. Smola, and Kostas Tsioutsiouliklis. 2012. Discovering geographical topics in the Twitter stream. In Proc. of $W W W$.

Yohei Ikawa, Miki Enoki, and Michiaki Tatsubori. 2012. Location inference using microblog messages. In Proc. of $W W W$.
Dekang Lin. 1998. Automatic retrieval and clustering of similar words. In Proc. of COLING-ACL.

Tomas Mikolov, Kai Chen, Greg Corrado, and Jeffrey Dean. 2013. Efficient estimation of word representations in vector space. In Proc. of ICLR.

Frederic Morin and Yoshua Bengio. 2005. Hierarchical probabilistic neural network language model. In Robert G. Cowell and Zoubin Ghahramani, editors, Proc. of AISTATS.

Brendan O'Connor, Jacob Eisenstein, Eric P. Xing, and Noah A. Smith. 2010. Discovering demographic language variation. In NIPS Workshop on Machine Learning and Social Computing.

Sharon Oviatt. 2003. Multimodal interfaces. In Julie A. Jacko and Andrew Sears, editors, The Human-computer Interaction Handbook, pages 286-304, Hillsdale, NJ, USA. L. Erlbaum Associates Inc.

Marco Pennacchiotti and Ana-Maria Popescu. 2011. Democrats, Republicans and Starbucks afficionados: User classification in Twitter. In Proc. of KDD.

Delip Rao, David Yarowsky, Abhishek Shreevats, and Manaswi Gupta. 2010. Classifying latent user attributes in Twitter. In Proc. of the Workshop on Search and Mining User-generated Contents.

Maya Ravindranath. 2011. A wicked good reason to study intensifiers in New Hampshire. In NWAV 40.

Joseph Reisinger and Raymond J. Mooney. 2010. Multi-prototype vector-space models of word meaning. In Proc. of NAACL.

Stephen Roller and Sabine Schulte im Walde. 2013. A multimodal LDA model integrating textual, cognitive and visual modalities. In Proc. of EMNLP.

Stephen Roller, Michael Speriosu, Sarat Rallapalli, Benjamin Wing, and Jason Baldridge. 2012. Supervised text-based geolocation using language models on an adaptive grid. In Proc. of EMNLP-CoNLL.

Richard Socher, Jeffrey Pennington, Eric H. Huang, Andrew Y. Ng, and Christopher D. Manning. 2011. Semi-supervised recursive autoencoders for predicting sentiment distributions. In Proc. of EMNLP.

Richard Socher, John Bauer, Christopher D. Manning, and $\mathrm{Ng}$ Andrew Y. 2013. Parsing with compositional vector grammars. In Proc. of $A C L$.

Joseph Turian, Lev Ratinov, and Yoshua Bengio. 2010. Word representations: A simple and general method for semi-supervised learning. In Proc. of ACL.

Peter D. Turney and Patrick Pantel. 2010. From frequency to meaning: Vector space models of semantics. Journal of Artificial Intelligence Research, 37(1):141-188, January. 
Benjamin P. Wing and Jason Baldridge. 2011. Simple supervised document geolocation with geodesic grids. In Proc. of ACL.

Chen Yu and Dana H. Ballard. 2004. A multimodal learning interface for grounding spoken language in sensory perceptions. ACM Transactions on Applied Perception, 1(1):57-80. 